Pacific Journal of Mathematic 


\title{
INDEPENDENCE OF NORMAL WEIERSTRASS POINTS UNDER DEFORMATION
}

\author{
R. F. LAX
}

Let $X$ denote a compact Riemann surface of genus $g$ and suppose $P \in X$. The Weierstrass nongaps at $P$ are those positive integers $n$ such that there exists a meromorphic function on $X$ which has a pole of order $n$ at $P$ and is holomorphic everywhere else. The Weierstrass semigroup at $P$, which we denote by $\Gamma(P)$, is the additive semigroup consisting of 0 and the nongaps. $A$ point $P$ is a Weierstrass point if there exists a nongap less than $g+1$ at $P$ and is a normal Weierstrass point if $\Gamma(P)=\{0, g, g+2, g+3, g+4, \cdots\}$. We consider here the following problem: Given a collection $P_{1}, \cdots, P_{n}$ of points on $X$, describe the infinitesimal variations of complex structure on $X$ which preserve the Weierstrass semigroups at $P_{1}, \cdots, P_{n}$. Our main result says, roughly speaking, that normal Weierstrass points deform as independently of each other as possible.

1. Let $T_{g}$ denote the Teichmuller space for Teichmuller surfaces of genus $g>1$ and let $\pi: V \rightarrow T_{g}$ denote the universal curve of genus $g$. Let $\mathscr{Y}_{k}^{r}$, for $k=2, \cdots, 2 g-2$ and $r=1,2, \cdots$, denote the closed complex subspaces of $V$ of Weierstrass points of the universal curve which were defined in [1], [2]. These spaces may be described set-theoretically as follows:

1) for $k \leqq g$, then

$\left|\mathscr{W}_{k}^{r}\right|=\left\{(t, P) \in V\right.$ : in the Weierstrass gap sequence at $P \in V_{t}$, there are at least $r$ nongaps $\leqq k\}$.

2) For $k \geqq g$, then

$\left|\mathscr{V}_{k}^{r}\right|=\left\{(t, P) \in V:\right.$ in the Weierstrass gap sequence at $P \in V_{t}$, there are at least $r$ gaps $>k\}$.

If $P$ is a point on a compact Riemann surface, let $\Gamma(P)$ denote the semigroup of Weierstrass nongaps at $P$. Let $\Gamma$ be an additive subsemigroup of the nonnegative integers and suppose $\Gamma$ has $g$ gaps.

Definition. Put $\mathscr{W}(\Gamma)=\{(t, P) \in V: \Gamma(P)=\Gamma\}$.

It is not hard to see that $\mathscr{\mathscr { V }}(\Gamma)$ is a (possibly empty) open subset of a finite intersection of $\mathscr{Y}_{k}^{r}$ 's. Thus $\mathscr{W}(\Gamma)$ has the structure of a complex analytic subspace of $V$.

Suppose $(t, P) \in V$ and put $X=V_{t}$. Let $c_{1}, \cdots, c_{3 g-3}$ denote Patt's local coordinates [3] on $T_{g}$ centered at $t$. These coordinates arise as variation parameters used in performing local variations of structure around $3 g-3$ generally chosen points on $X$. Let $z$ be a 
local coordinate on $X$ centered at $P$. Let $1<\gamma_{2}<\cdots<\gamma_{g}$ denote the Weierstrass gaps at $P$ and choose a basis $d \zeta_{1}, \cdots, d \zeta_{g}$ of holomorphic differentials on $X$ such that $\operatorname{ord}_{p} d \zeta_{j}=\gamma_{j}-1, j=1, \cdots, g$. Write $d \zeta_{j}=f_{j}(z) d z$.

We next describe coordinates for $T_{(t, P)}(V)$, the tangent space to $V$ at $(t, P)$. We may view a tangent vector $\xi$ to $V$ at $(t, P)$ as a $C$-homomorphism of local rings, $\xi: \mathcal{O}_{V,(t, P)} \rightarrow C[\varepsilon] /\left(\varepsilon^{2}\right)$. Then $\xi$ is determined by its values on a set of local parameters of $\mathscr{O}_{V,(t, P)}$. So, if $\xi(t)=u \varepsilon, \xi\left(c_{m}\right)=b_{m} \varepsilon, m=1, \cdots, 3 g-3$, then $\left(u, b_{1}, \cdots, b_{3 g-3}\right)$ serve as coordinates for $T_{(t, P)}(V)$. From [1], we have

Proposition 1. Suppose $(t, P) \in \mathscr{W}_{k}^{r}$. Then $\xi \in T_{(t, p)}\left(\mathscr{W}_{k}^{r}\right)$ if and only if all minors of order $\rho-r+1$, where $\rho=\min (k, g)$, of the matrix

$$
M(t, P)=\left[\begin{array}{l:c}
f_{j}^{(i)}(0) & \varepsilon\left(u \cdot f_{j}^{(i+1)}(0)+(i !) \sum_{m=1}^{3 g-3} b_{m} \tau_{P, i}^{\prime}\left(Q_{m}\right) \zeta_{j}^{\prime}\left(Q_{m}\right)\right) \\
i=0, \cdots, k-1 & i=0, \cdots, k-1 \\
j=1, \cdots, \rho-r & j=\rho-r+1, \cdots, g
\end{array}\right]
$$

vanish, where $\tau_{P, \ell}$ is an elementary integral of the second kind on $X$ with pole of order $\ell+1$ at $P$ and where $\left(Q_{1}, \cdots, Q_{3 g_{-3}}\right)$ is any point chosen from an open subset of $X^{3 g-3}$.

The vanishing of each minor of order $\rho-r+1$ of the above matrix gives a linear equation in $u, b_{1}, \cdots, b_{3 g-3}$ and these equations cut out $T_{(t, P)}\left(\mathscr{W}_{k}^{r}\right)$ as a linear subspace of $T_{(t, P)}(V)$. These equations are of the form $E_{n}: a_{n} u+\sum_{m=1}^{s g-3} \beta_{n}\left(Q_{m}\right) b_{m}=0$, where the $\beta_{n}$ are quadratic differentials, possibly with a pole at $P$, on $X$ (arising from the products $d \tau_{P, \ell} d \zeta_{j}$ which occur in $M$ ) and where by $\beta_{n}\left(Q_{m}\right)$ we mean $\beta_{n} /\left(d z_{m}\right)^{2}(0)$ with $z_{m}$ being a local coordinate on $X$ centered at $Q_{m}$.

We note that if $(t, P) \in \mathscr{W}_{k}^{r}-\mathscr{Y}_{k}^{r+1}$, then $M$ will have a nonzero minor of order $\rho-r$, call it $\mu$, which comes from the first $\rho-r$ columns. In order that all minors of order $\rho-r+1$ vanish, it is sufficient that only those minors of order $\rho-r+1$ which contain $\mu$ should vanish. This gives rise to $r(|k-g|+r)$ linear equations in $u, b_{1}, \cdots, b_{3 g-3}$.

Now, since $\mathscr{W}(\Gamma(P))$ is an open subspace of an intersection of $\mathscr{W}_{k}^{r}$ 's, the tangent space to $\mathscr{W}^{\prime}(\Gamma(P))$ at $(t, P)$ is cut out as a linear subspace of $T_{(t, P)}(V)$ by a collection of linear equations of the above form. Put

$$
Y(P)=T_{(t, P)}(\mathscr{W}(\Gamma(P))) \cap\{u=0\} .
$$


We may view $Y(P)$ as a linear subspace of $T_{t}\left(T_{g}\right)$ via identifying the subspace $\{u=0\}$ of $T_{(t, P)}(V)$ with $T_{t}\left(T_{g}\right)$. One may think of $Y(P)$ as being the space of infinitesimal deformations of $X$ which leave the semigroup of Weierstrass nongaps at $P$ unchanged.

In some special cases, we know the dimension of $Y(P)$. For example, the next proposition follows from the description of $T_{(t, p)}\left(\mathscr{W}_{g+\ell-1}^{1}\right)$ in [2].

Proposition 2. Suppose that the Weierstrass gap sequence at $P \in X$ is $1,2, \cdots, g-1, g+\ell$, where $1 \leqq \ell \leqq g-1$. Then $\operatorname{dim} Y(P)=$ $3 g-3-\ell$. Thus, the family of infinitesimal deformations of $X$ which leave the Weierstrass semigroup at $P$ unchanged has codimension $<$ in the family of all infinitesimal deformations of $X$.

Definition. Suppose $P_{1}, \cdots, P_{n}$ are Weierstrass points on $X$. We will say that $P_{1}, \cdots, P_{n}$ are infinitesimally in general position if $Y\left(P_{1}\right), \cdots, Y\left(P_{n}\right)$ are in general position as linear subspaces of $T_{t}\left(T_{g}\right)$. (By this we mean that if $\left\{Y\left(P_{i}\right)\right\}_{i \in I}$ is any subset of $\left\{Y\left(P_{1}\right), \cdots, Y\left(P_{n}\right)\right\}$, then these linear subspaces of $T_{t}\left(T_{g}\right)$ may be cut out by a collection of linear equations such that any subset of these equations has maximum rank.)

To illustrate this concept, we will consider hyperelliptic curves of genera 2 and 3 in the next section.

\section{2.}

Proposition 3. The Weierstrass points of a compact Riemann surface of genus 2 are infinitesimally in general position.

Proof. Suppose $X=V_{t}$ is the compact Riemann surface defined by the equation $y^{2}=\prod_{i=1}^{6}\left(x-a_{i}\right)$ and let $P_{i}$ denote the branch point over $a_{i}, i=1, \cdots, 6$. It is well-known that $P_{1}, \cdots, P_{6}$ are the Weirstrass points of $x$.

Note that $\mathscr{W}\left(\Gamma\left(P_{i}\right)\right)=\mathscr{\mathscr { N }}_{2}^{1}$. We compute $T_{\left(t, P_{i}\right)}\left(\mathscr{W}_{2}^{1}\right)$. As basis for the holomorphic differentials on $X$, we take $d \zeta_{1}=y^{-1} d x, d \zeta_{2}=$ $\left(x-a_{i}\right) y^{-1} d x$. Let $d \tau_{i, \ell}$ denote the normalized elementary differential of the second kind on $X$ with pole of order $\ell+2$ at $P_{i}$. Then the matrix $M\left(t, P_{i}\right)$ of $\S 1$ is

$$
M\left(t, P_{i}\right)=\left[\begin{array}{ll}
d_{1} & \varepsilon \sum_{m=1}^{3} b_{m} \tau_{i, 0}^{\prime}\left(Q_{m}\right) \zeta_{2}^{\prime}\left(Q_{m}\right) \\
0 & \varepsilon\left(d_{2} u+\sum_{m=1}^{3} b_{m} \tau_{i, 1}^{\prime}\left(Q_{m}\right) \zeta_{2}^{\prime}\left(Q_{m}\right)\right.
\end{array}\right]
$$

where $d_{1}, d_{2}$ are nonzero complex numbers. Then it is easy to see 
that $Y\left(P_{i}\right)=\left\{\left(b_{1}, b_{2}, b_{3}\right): \sum_{m=1}^{3} \beta_{i}\left(Q_{m}\right) b_{m}=0\right\}$ where $\beta_{i}=d \tau_{i, 1} d \zeta_{2}$. Now, since $\beta_{i}$ has a pole at $P_{i}$ and is finite at $P_{j}, i \neq j$, we have that $\beta_{1}, \cdots, \beta_{6}$ are linearly independent. Then, since $\left(Q_{1}, Q_{2}, Q_{3}\right)$ is any point from an open subset of $X^{3}$, it is not hard to see that the hyperplanes $\sum_{m=1}^{3} \beta\left(Q_{i}\right) b_{m}=0, i=1, \cdots, 6$, will be in general position in $T_{t}\left(T_{2}\right)$.

The proposition is intuitively clear if one views deformations of a Riemann surface of genus 2 as arising from variations of the branch points of the two-sheeted convering of the Riemann sphere. One can certainly vary these branch points independently (although there is only a three-dimensional family of independent variations) and the Weierstrass points are just these branch points.

Now suppose $X$ is a hyperelliptic Riemann surface of genus 3 and let $P_{1}, \cdots, P_{8}$ denote the Weierstrass points on $X$. By the above reasoning, one should expect that $Y\left(P_{1}\right), \cdots, Y\left(P_{8}\right)$ will be in general position as subspaces of the space of infinitesimal deformations of $X$ which preserve hyperellipticity, and so these spaces will not be in general position as subspaces of the tangent space to $T_{3}$ at the point corresponding to $X$. Intuitively, if $P_{1}$ is to remain a hyperelliptic Weierstrass point, then a condition is imposed on $P_{2}, \cdots, P_{8}$, namely that they cannot deform to normal Weierstrass points.

We can indicate what should be occurring in the matrices $M\left(t, P_{i}\right)$ at two points on a hyperelliptic Riemann surface of genus 3. Suppose $X=V_{t}$ is defined by the equation $y^{2}=\prod_{i=1}^{8}\left(x-a_{i}\right)$ and that $P_{1}$ and $P_{2}$ are the branch points over $a_{1}$ and $a_{2}$ respectively. Let $d \tau_{i, \ell}$ denote the normalized elementary differential of the second kind with pole of order $\ell+2$ at $P_{1}$.

To compute $T_{\left(t, P_{1}\right)}\left(\mathscr{W}_{2}^{1}\right)$, take $d \zeta_{1,1}=y^{-1} d x, d \zeta_{1,2}=\left(x-a_{1}\right) y^{-1} d x$, $d \zeta_{1,3}=\left(x-a_{1}\right)^{2} y^{-1} d x$. Then the matrix $M\left(t, P_{1}\right)$ will be

$$
\left[\begin{array}{lll}
d_{1} & \varepsilon\left(\sum_{m=1}^{6} b_{m} \tau_{1,0}^{\prime}\left(Q_{m}\right) \zeta_{1,2}^{\prime}\left(Q_{m}\right)\right) & \varepsilon\left(\sum_{m=1}^{6} b_{m} \tau_{1,0}^{\prime}\left(Q_{m}\right) \zeta_{1,3}^{\prime}\left(Q_{m}\right)\right) \\
0 & \varepsilon\left(d_{2} u+\sum_{m=1}^{6} b_{m} \tau_{1,1}^{\prime}\left(Q_{m}\right) \zeta_{1,2}^{\prime}\left(Q_{m}\right)\right) & \varepsilon\left(\sum_{m=1}^{6} b_{m} \tau_{1,1}^{\prime}\left(Q_{m}\right) \zeta_{1,3}^{\prime}\left(Q_{m}\right)\right)
\end{array}\right]
$$

where $d_{1}, d_{2}$ are nonzero complex numbers. Hence $Y\left(P_{1}\right)$ will be $\left\{\left(b_{1}, \cdots, b_{6}\right): \sum_{m=1}^{6} \beta_{1,1}\left(Q_{m}\right) b_{m}=0\right.$ and $\left.\sum_{m=1}^{6} \beta_{1,2}\left(Q_{m}\right) b_{m}=0\right\}$, where $\beta_{1,1}=$ $d \tau_{1,1} d \zeta_{1,2}$ and $\beta_{1,2}=d \tau_{1,1} d \zeta_{1,3}$.

Similarly, taking $d \zeta_{2,1}=y^{-1} d x, d \zeta_{2,2}=\left(x-a_{2}\right) y^{-1} d x, d \zeta_{2,3}=(x-$ $\left.a_{2}\right)^{2} y^{-1} d x$ and computing $M\left(t, P_{2}\right)$, one finds that $Y\left(P_{2}\right)=\left\{\left(b_{1}, \cdots, b_{6}\right)\right.$ : $\sum_{m=1}^{6} \beta_{2,1}\left(Q_{m}\right) b_{m}=0$ and $\left.\sum_{m=1}^{6} \beta_{2,2}\left(Q_{m}\right) b_{m}=0\right\}$, where $\beta_{2,1}=d \tau_{2,1} d \zeta_{2,2}$ and $\beta_{2,2}=d \tau_{2,1} d \zeta_{2,3}$.

Now $\beta_{1,1}$ has a simple pole at $P_{1}$ and $\beta_{2,1}$ has a simple pole at $P_{2}$, but $\beta_{1,2}$ and $\beta_{2,2}$ are finite everywhere. Indeed, if $\left(x-a_{i}\right)^{-2} d x$ 
were the normalized elementary differential of the second kind with pole of order 3 at $P_{i}$, then we would have $\beta_{1,2}=\beta_{2,2}=y^{-1}(d x)^{2}$ and the two 4-planes $Y\left(P_{1}\right)$ and $Y\left(P_{2}\right)$ would intersect in a 3-plane. Unfortunately, it seems that one must know these normalized elementary differentials of the second kind explicitly to be able to perfect this argument.

3. We now prove our main result, which generalizes Proposition 3.

THEOREM. Let $X$ be a compact Riemann surface of genus $g>1$. Suppose $P_{1}, \cdots, P_{n}$ are normal Weierstrass points on $X$ and $Q$ is any other Weierstrass point on $X$. Then $P_{1}, \cdots, P_{n}, Q$ are infinitesimally in general position.

Proof. Let $P$ denote a normal Weierstrass point on $X$. Then $\mathscr{W}(\Gamma(P))=\mathscr{W}_{g}^{1}-\left(\mathscr{W}_{g-1}^{1} \cup \mathscr{W}_{g+1}^{1}\right)$. We compute $T_{(t, P)}\left(\mathscr{W}_{g}^{1}\right)$, where $X=V_{t}$.

With notation as in $\S 1$, the matrix $M(t, P)$ will be

$$
\left[\begin{array}{l:c}
f_{j}^{(i)}(0) & \varepsilon\left(u \cdot f_{g}^{(i+1)}(0)+(i !) \sum_{m=1}^{3 g-3} b_{m} \tau_{P, i}^{\prime}\left(Q_{m}\right) \zeta_{g}^{\prime}\left(Q_{m}\right)\right) \\
i=0, \cdots, g-1 & i=0, \cdots, g-1 \\
j=1, \cdots, g-1 &
\end{array}\right] .
$$

Let $\beta_{P}\left(Q_{m}\right)$ denote the coefficient of $b_{m}$ in the equation $\operatorname{det} M(t, P)=$ 0 . Expanding $\operatorname{det} M(t, P)$ by its last row, it is easy to see that $\beta_{P}$ will have a nonzero term in $d \tau_{P, g-1} d \zeta_{g}$ and thus the order of $\beta_{P}$ at $P$ will be $-(g-1+2)+g=-1$. Performing this analysis at $P_{1}, \cdots, P_{n}$, we see that if $\beta_{P_{2}}\left(Q_{m}\right)$ denotes the coefficient of $b_{m}$ in the equation $\operatorname{det} M\left(t, P_{i}\right)=0$, then $\beta_{P_{i}}$ will have a pole at $P_{i}$ and be finite elsewhere.

Now suppose that $Y(Q)$ is cut out as a subspace of $T_{t}\left(T_{g}\right)$ by linearly independent equations $E_{1}, \cdots, E_{k}$, where $E_{j}$ is of the form $\sum_{m=1}^{3 g-3} \alpha_{j}\left(Q_{m}\right) b_{m}=0$, as in $\S 1$. Then $\alpha_{1}, \cdots, \alpha_{k}$ will be linearly independent and will be finite at $P_{1}, \cdots, P_{n}$. Hence, $\beta_{P_{1}}, \cdots, \beta_{P_{n}}, \alpha_{1}, \cdots, \alpha_{k}$ are linearly independent quadratic differentials and $Y\left(P_{1}\right), \cdots, Y\left(P_{n}\right)$, $Y(Q)$ will be in general position as subspaces of $T_{t}\left(T_{g}\right)$.

COROLLARY. The normal Weierstrass points on a compact Riemann surface are infinitesimally in general position.

The question of when nonnormal Weierstrass points are infinitesimally in general position remains open. 


\section{REFERENCES}

1. R. F. Lax, Weierstrass points of the universal curve, Math. Ann., 216 (1975), 35-42.

2. —_, Gap sequences and moduli in genus four, Math. Z., 175 (1980), 67-75.

3. C. Patt, Variations of Teichmuller and Torelli surfaces, J. Analyse Math., 11 (1963), 221-247.

Received December 28, 1980 and in revised form February 25, 1981.

Louisiana State University

Baton Rouge, LA 70803 


\section{PACIFIC JOURNAL OF MATHEMATICS}

\section{EDITORS}

DONALD BABBITT (Managing Editor)

University of California

Los Angeles, CA 90024

Hugo Rossi

University of Utah

Salt Lake City, UT 84112

C. C. MOore and Arthur Agus

University of California

Berkeley, CA 94720
J. DugundJI

Department of Mathematics

University of Southern California

Los Angeles, CA 90007

R. FinN and J. MILGRAM

Stanford University

Stanford, CA 94305

\section{ASSOCIATE EDITORS}
R. ARENS
E. F, BECKENBACH
B. H. NeumanN
F. WOLF
K. YoSHIDA

\section{SUPPORTING INSTITUTIONS}

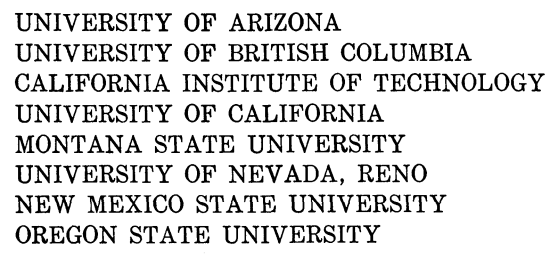

UNIVERSITY OF ARIZONA

UNIVERSITY OF BRITISH COLUMBIA

CALIFORNIA INSTITUTE OF TECHNOLOGY

UNIVERSITY OF CALIFORNIA

MONTANA STATE UNIVERSITY

UNIVERSITY OF NEVADA, RENO

NEW MEXICO STATE UNIVERSITY

OREGON STATE UNIVERSITY

\author{
UNIVERSITY OF OREGON \\ UNIVERSITY OF SOUTHERN CALIFORNIA \\ STANFORD UNIVERSITY \\ UNIVERSITY OF AAWAII \\ UNIVERSITY OF TOKYO \\ UNIVERSITY OF UTAH \\ WASHINGTON STATE UNIVERSITY \\ UNIVERSITY OF WASHINGTON
}

The Supporting Institutions listed above contribute to the cost of publication of this Journal, but they are not owners or publishers and have no responsibility for its content or policies,

Mathematical parers intended for publication in the Pacific Journal of Mathematics should be in typed form or offset-reproduced, (not dittoed), double spaced with large margins. Please do not use built up fractions in the text of the manuscript. However, you may use them in the displayed equations. Underline Greek letters in red, German in green, and script in blue. The first paragraph or two must be capable of being used separately as a synopsis of the entire paper. Please propose a heading for the odd unmbered pages of less than 35 characters. Manuscripts, in triplicate, may be sent to any one of the editors. Please classify according to the scheme of Math. Reviews, Index to Vol. 39. Supply name and address of author to whom proofs should be sent. All other communications should be addressed to the managing editor, or Elaine Barth, University of California, Los Angeles, California, 90024.

50 reprints to each author are provided free for each article, only if page charges have been substantially paid. Additional copies may be obtained at cost in multiples of 50 .

The Pacific Journal of Mathematics is issued monthly as of January 1966, Regular subscription rate: $\$ 114.00$ a year $(6$ Vol., 12 issues). Special rate: $\$ 57.00$ a year to individual members of supporting institution.

Subscriptions, orders for numbers issued in the last three calendar years, and changes of address shoud be sent to Pacific Journal of Mathematics, P.O. Box 969, Carmel Valley, CA 93924, U.S.A. Old back numbers obtainable from Kraus Periodicals Co., Route 100, Millwood, NY 10546.

PUBLISHED BY PACIFIC JOURNAL OF MATHEMATICS, A NON-PROFIT CORPORATION

Printed at Kokusai Bunken Insatsusha (International Academic Printing Co., Ltd.). 8-8, 3-chome, Takadanobaba, Shinjuku-ku, Tokyo 160, Japan.

Copyright (C) 1982 by Pacific Journal of Mathematics Manufactured and first issued in Japan 


\section{Pacific Journal of Mathematics}

\section{Vol. 101, No. 2 December, 1982}

Jean Bourgain, A Hausdorff-Young inequality for $B$-convex Banach

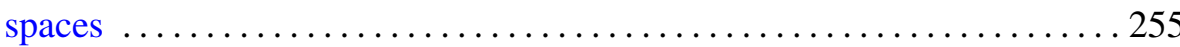

J. L. Brenner and Lorraine L. Foster, Exponential Diophantine

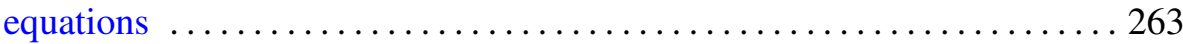

Henry H. Glover and William Duncan Homer, II, Fixed points on flag

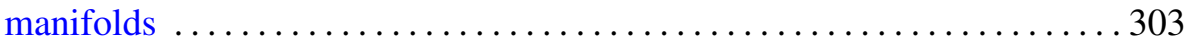

Lothar Hahn, A note on stochastic methods in connection with approximation theorems for positive linear operators $\ldots \ldots \ldots \ldots \ldots . \ldots 307$

James P. Henderson, Approximating cellular maps between low-dimensional polyhedra

V. K. Jain, Certain transformations of basic hypergeometric series and their

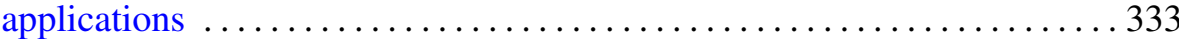

Charles David Keys, On the decomposition of reducible principal series

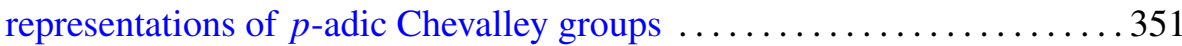

M. S. Klamkin and A. Meir, Ptolemy's inequality, chordal metric,

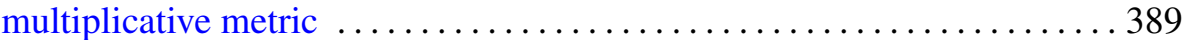

Robert F. Lax, Independence of normal Weierstrass points under

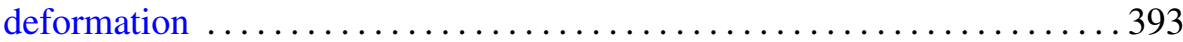

Leonid A. Luxemburg, On compactifications of metric spaces with

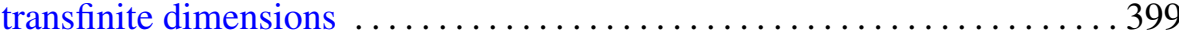

Carlton James Maxson, Martin Ross Pettet and Kirby C. Smith, On semisimple rings that are centralizer near-rings .....

Teodor C. Przymusiński, Extending functions from products with a metric factor and absolutes

Giorgio Talenti, A note on the Gauss curvature of harmonic and minimal surfaces

D. M. Terlinden, A spectral containment theorem analogous to the semigroup theory result $e^{t \sigma(A)} \subseteq \sigma\left(e^{t A}\right)$ 